\title{
Establishing an emergency nurse practitioner service
}

J Bache

The role of the nurse in the emergency department has evolved over recent years. ${ }^{1}$ Emergency nurse practitioners (ENPs) were introduced into the accident and emergency (A\&E) department at Leighton Hospital in July 1992, when designated nurse practitioner schemes were rare. ${ }^{2}$ They have been of enormous benefit to the patients and staff since then. This paper describes some of the potential advantages and disadvantages of an ENP service, and provides some practical advice on establishing such a service.

Potential advantages of an ENP service Virtually all A\&E departments in the United Kingdom perceive themselves to be understaffed, particularly as far as medical staffing is concerned. An intrinsic difficulty in establishing adequate staffing levels is the variable and largely unpredictable nature of the workload. There are more A\&E nurses than doctors, so that sharing some of the workload between the two professions will result in greater flexibility. $\mathrm{A} \& \mathrm{E}$ is an intensely practical specialty and it seems illogical to suggest that a senior house officer (SHO), new to A\&E, will be more knowledgeable than a nurse with years of experience, particularly when considering conditions which, in hospital practice, are virtually exclusive to $\mathrm{A} \& \mathrm{E}$, such as sprains, minor cuts and bruises, and minor head injuries. $A \& E$ is very much a team effort and lends itself to the team approach. ${ }^{3}$ Perceived benefits of providing an ENP service identified by Tye et al included improved waiting times, increased job satisfaction for ENPs, improved quality of care, increased patient satisfaction, better use of resources, more holistic care, and improved continuity of care. ${ }^{4}$

Consequently, it seems reasonable to suggest that experienced nurses, after adequate training, will be able to share the workload of the doctors and treat suitable patients independently without reference to the doctors. Suitable nurses will be able to expand their role and increase the interest of their work, while helping their medical colleagues and reducing the waiting time in the department. Sakr et al have demonstrated that properly trained $A \& E$ nurse practitioners, working within agreed guidelines, can provide care for patients with minor injuries that is equal to or in some ways better than that provided by junior doctors. ${ }^{5}$ Crux found that $83 \%$ of patients visiting the A\&E department at the Royal Victoria Hospital in Folkestone could have been treated by an ENP. ${ }^{6}$ Brebner et al, however, found that ENPs could have examined, treated and discharged $33 \%$ of the total throughput in the A\&E department at Aberdeen Royal Infirmary from 900 am to $900 \mathrm{pm} .^{7}$

\section{Potential disadvantages of an ENP service}

Perceived problems of providing an ENP service identified by Tye et al included staffing difficulties, opposition from other professional groups, funding, limitation of protocols, and medicolegal concerns. ${ }^{4}$ The service can become so successful that the department becomes dependent upon the ENPs, to the extent that a clinical service cannot be provided in the absence of ENPs. Consequently, medical staffing levels remain inadequate because ENPs are undertaking a considerable proportion of the workload. However, ENPs are not able to manage all the patients who need to be seen by medical staff. Therefore, if the proportion of patients needing to be seen by medical staff is high, either permanently or at any particular time, medical staffing levels may be inadequate.

For similar reasons, the ENPs may be seeing so many patients in their own right that they rarely have the opportunity to perform the more traditional functions of senior nursing staff in A\&E, such as assisting at resuscitations and in the management of major trauma. They may become resentful of the perceived loss of these important aspects of their role, and may eventually feel apprehensive and even incapable of managing such situations. They may feel that the SHOs are taking advantage of their skills, and working less hard. Conversely, the SHOs may feel that they are expected to see all the major problems, such as the collapses, while the ENPs are treating the more minor and easier conditions.

\section{Hints on establishing an ENP service}

1 SELECT SUITABLE NURSES

The five main qualities required are experience; common sense; the ability to accept responsibility; the ability to recognise limitations; and the ability to ask for help. It is illogical to specify a required length of experience since qualification or in $\mathrm{A} \& \mathrm{E}$, but clearly it is essential to have a good breadth of experience. Common sense is a quality present in abundance in most A\&E staff, almost all of whom are comfortable in accepting responsibility and are able to recognise their limitations. These essential qualities correlate well with the qualities required of any good $A \& E$ nurse. The ability to recognise their limitations is clearly related to the ability to ask for help, which is in turn intimately related to the approachability of the medical staff. All ENPs in Leighton Hospital are $\mathrm{G}$ grade nurses. 
2 DEFINE THE CONTENTS OF THE ROLE

This should be done by mutual agreement between the selected nurses and the senior medical staff. The nurses must become confident in their role and the medical staff must share in that confidence and feel that the intended role will be beneficial. Precise details of the role may vary not only from department to department but also from ENP to ENP, and may change as their experience changes. Suitable conditions for treatment by ENPs may include abrasions, bruises, sprains, certain lacerations, bites and stings, minor burns and scalds, and minor head injuries.

Particularly important decisions include whether the ENPs can order and/or interpret certain radiographs; and which medications, if any, the ENPs are allowed to prescribe. The role of the ENP is enormously improved by the ability to order and/or interpret certain radiographs and to prescribe certain drugs, but these can only be decided after detailed discussions with the radiologists and pharmacists. Meek et al investigated the ability of nurse practitioners in $\mathrm{A} \& \mathrm{E}$ to interpret distal limb radiographs and found that they were able to interpret the films to a standard equal to SHOs with three to five months' experience ${ }^{8}$; they found that those nurse practitioners actively interpreting radiographs as part of their role in minor injury units were able to interpret the films to the same standard as SHOs with more that five months' experience. Salt and Clancy demonstrated that nurses can apply the Ottawa ankle rules safely without missing acute fractures. ${ }^{9}$ Mann et al showed that nurse practitioners trained to use the Ottawa ankle rules are as effective as SHOs in the implementation of the rules. ${ }^{10}$ Thurston and Field investigated whether waiting time in $\mathrm{A} \& \mathrm{E}$ departments is shortened when experienced nurses request peripheral limb radiographs before a patient is assessed by a doctor, and found that 14 minutes can be saved by arranging for the patient to see the nurse first, but nurses tended to request more radiological examinations than doctors, and they concluded that specific training and constant monitoring are essential. ${ }^{11}$ Freij et al compared the ability of nurse practitioners and SHOs to request and interpret a limited range of radiographic views of patients attending a minor injuries unit, and found that appropriately trained nurse practitioners are at least as good as SHOs in recognising the need for a radiograph and are as competent in their interpretation. ${ }^{12}$ Sakr et al did not find that nurse practitioners requested radiography more frequently than doctors; both groups interpreted the radiographs with equal accuracy. ${ }^{5}$ It is advisable, when establishing an ENP service, to conduct trials to ascertain how the proposals are likely to affect the workloads and the budgets of the relevant departments.

The hospital pharmacists will be aware of the legal implications of prescribing by nurses. Marshall et al investigated the legal and professional issues related to nurse administration of drugs according to protocols and concluded that there are no legal or professional obstacles to the development of protocols for the admin- istration of drugs to patients by nurses without reference to a doctor, providing the protocols meet all the requirements of the United Kingdom Central Council for Nursing, Midwifery and Health Visiting (UKCC) and have the support of consultant medical staff. ${ }^{13}$ They noted that such a system must be subject to regular audit to promote a dynamic approach to protocols and training.

3 DEFINE THE LIMITS OF THE ROLE

Conditions or situations which are considered inappropriate for treatment by an ENP are again determined by mutual agreement between the selected nurses and the senior medical staff. Obvious examples include patients with chest pain, major trauma, or trauma likely to require admission. Other possibilities include children under 1 year of age, victims of assaults, and victims of road traffic accidents. The limits are again determined by mutual agreement and must be fully understood and agreed by all the nursing and medical staff.

All patients should be informed that they have a right to be seen by a doctor if they wish, although we have found that this offer is rarely accepted.

4 COMMUNICATE WITH OTHER PROFESSIONALS Communication is essential to the successful introduction of ENPs. All personnel within the department must be kept informed of developments. Other staff who may be affected will also need to be included in discussions at all stages. Of particular importance are clinicians likely to receive referrals directly from the ENPs, such as the orthopaedic surgeons; radiologists and radiographers if the ENPs will be ordering radiographs; and pharmacists if the ENPs will be prescribing. Senior managers will also need to be involved in discussions, particularly the medical director, the director of nursing, and the chief executive. Local general practitioners will wish to be kept abreast of developments. It is advisable to describe the proposed changes in the local press.

\section{AGREE GUIDELINES AND PROTOCOLS}

Having decided which conditions are suitable for management by ENPs, guidelines and protocols must be agreed. Again it is essential to ensure that all interested parties are invited to contribute. The guidelines and protocols followed by the ENPs must be identical to those followed by the medical staff.

\section{TEACH THE APPROPRIATE SKILLS}

There is considerable variation in the nature and logistics of the teaching. A questionnaire distributed in January 1994 revealed that in 31 of 49 major departments with "official" nurse practitioners $(63 \%)$, the practitioners received formal training internally from a combination of doctors and nurses; in 15 of the 49 departments $(31 \%)$, practitioners were sent on an external course; practitioners in six of the 49 departments $(12 \%)$ claimed to have received no formal training at all. ${ }^{14}$ In a postal survey conducted in May 1996, Tye et al ${ }^{4}$ found that $91(93 \%)$ of the 98 major A\&E departments in 
the UK currently providing an ENP service employed ENPs who had received some form of education or training for the role; however, wide variations were evident: 55 departments $(60 \%)$ provided training inhouse, with 30 $(33 \%)$ receiving training from a relatively small number of external establishments. At one extreme, it has been suggested that a mastership is required.

When ENPs were introduced at Leighton Hospital, in July 1992, a training package was written and we arranged a training day that was devoted to the practical skills involved. This included lectures on topics such as note writing; anatomy and examination of the eye; anatomy and examination of the upper limb; anatomy and examination of the distal lower limb; wound management and suturing of lacerations; management of burns and scalds; and management of fractures and plaster techniques. A textbook of practical procedures is invaluable. ${ }^{15}$

Since the introduction of ENPs, similar training days have been arranged as new ENP posts have been established. Topics may be added or removed as necessary. It is essential that each potential ENP attends all the teaching sessions, regardless of previous experience, although some ENPs may require particular emphasis on certain topics. The day concludes with a test to ensure that the essential facts have been understood. Each potential ENP is encouraged to comment upon the perceived relevance of each topic.

7 BE APPROACHABLE

Nurses hitherto accustomed to a relatively traditional role may well feel apprehensive when they first assume responsibility for managing a patient in their own right. It is essential that all senior medical staff emphasise that they will be immediately available if the ENP is unsure about the management of a specific patient.

8 BE SUPPORTIVE

Like doctors, nurses can make mistakes. Such mistakes should be recognised, corrected, and regarded as learning opportunities. An open policy is essential, on the principle that we can all make mistakes and we should take the opportunity to learn from the mistakes of ourselves and our colleagues.

9 AUDIT PROGRESS

The work of the ENPs should be audited, together with that of the medical staff. Topics suitable for audit include missed fractures, rate of ordering radiographs, patient satisfaction, wound infections, prescribing and note keeping. All these have been audited at Leighton Hospital and have shown that ENPs are as effective as SHOs.

10 REVIEW PROGRESS

The functions of each ENP need to be reviewed at regular intervals, as part of their personal performance plans. Their repertoires can be varied from time to time, according to their individual interests and requirements, and the needs of the department. The role of the ENPs should be dynamic, rather than static.
11 MAINTAIN KNOWLEDGE An annual "ENP updating day" is essential. Treatments and fashions change with the passage of time and it is easy to become locked in a time warp if a conscious effort is not made to avoid this. An annual assessment is required to ensure that competence is maintained.

12 MAINTAIN TRADITIONAL ROLE

It would be very easy for ENPs to become exclusively involved in the treatment of "minor injuries", particularly in a department with poor levels of medical staffing. Senior A\&E nurses enjoy managing major trauma and other serious medical conditions and it is essential that they do not lose these skills by concentrating on their roles as ENPs. Robinson and Inyang note that concerns about the loss of clinical judgement and skills because of specialisation can be resolved by rotation of nurse practitioner duties with those of traditional nursing. ${ }^{1}$

\section{ENCOURAGE DISCUSSION}

Teamwork is essential in A\&E and ENPs rapidly become invaluable members of the team. As such, it is essential that they contribute to future plans for the department.

\section{SEEK NEW BLOOD}

Potential ENPs should be sought, their skills should be developed, and they should be encouraged to apply for ENP posts at a suitable stage of their careers.

15 DECIDE HOW THE SERVICE WILL ACTUALLY FUNCTION

Flexibility is the key to success. ${ }^{16}$ When an ENP comes on duty at Leighton Hospital, they are not designated as either an ENP or a sister/ charge nurse for that shift. Instead, their function can change from minute to minute or hour to hour, according to the case mix in the department at any one time. If there are two patients with myocardial infarctions and three seriously injured in a road traffic accident, the ENP is likely to assume the traditional role of a senior A\&E nurse. If, an hour later, there is an influx of patients with relatively minor injuries, the same individual will function as an ENP. Tye et $a l^{4}$ undertook a postal survey of senior nurses of all major A\&E departments in the UK in May 1996 ( $n=293)$, and 98 ENP services were reported operational in major $A \& E$ departments. Three different operational models were identified. Twenty six departments $(27 \%)$ reported using a dedicated role approach in which the ENPs were permanently employed in that capacity and did not take on any other duties. Fifty three departments $(54 \%)$ had developed an integrated model, in which the role was combined with other nursing duties, often on an ad hoc basis. Fourteen (14\%) made the role operational on a rotational basis, with the ENPs rostered in that specific capacity for a defined period, after which they returned to their conventional role. Five departments failed to specify which approach they adopted. 
16 CONSIDER THE FUTURE

Future developments depend upon the individuals involved, the ability of senior staff to introduce change and progress, and government policy. For example, ENPs will clearly have a major part to play in the successful establishment of minor injury units and the introduction of telemedicine. Mabrook and Dale $^{17}$ demonstrated that ENPs can provide a useful service in a minor injuries unit based in a community hospital situated eight miles from its sister district hospital; nurses with a minimum of five years' $\mathrm{A} \& \mathrm{E}$ experience were appointed and they attended a formal three month ENP course; they could request and interpret certain radiographs and prescribe certain medications, including antibiotics.

A nationally recognised ENP course would ensure the required knowledge and expertise, and this would increase the confidence of new ENPs. At present there is no agreed syllabus or national accreditation for nurse practitioner courses. ${ }^{1}$ There is, at present, a great variety of methods of training, the academic content and extent of which varies enormously. At one extreme, ENPs can be trained inhouse, as we have successfully done at Leighton Hospital. This training can be supplemented, for example, by one day courses on radiographic interpretation, which are available at Hope Hospital in Salford. The nurse practitioners in the study by Sakr et a $\bar{P}$ were all senior nurses with at least four years' experience of working in an A\&E department. They were all working towards the qualification of A33, development of autonomous practice, from the English National Board. The clinical course consists of three weeks of theory, and includes anatomy, physiology, examination skills, interpretation of radiographs, and suturing. Each nurse then has 35 days of supervised clinical practice and has to pass a competency based assessment. The division of post-registration nursing at the University of Hertfordshire runs an ENP course (ENB A33), which lasts for a total of 15 days, in three five-day block releases over approximately three months. The department of emergency medicine at Hope Hospital in Salford also offers an ENP module (R34). The ENP course at Southend Hospital lasts for 40 hours over five days and consists of theoretical, practical and self directed study. Southend Hospital also offers a radiological interpretation course for ENPs, which lasts for 16 hours over two days. The autonomous health care practice course is a generic course offered to a range of health care professionals who are expanding their roles, to increase knowledge and clinical skills in areas normally considered to be the province of medicine. ${ }^{18}$

Tye has explored the literature concerning the development of the ENP role and has highlighted the current inconsistencies in educational provision, contrasting the relative plethora of rigorous evaluation studies in North America with the current paucity of empirical data available in the UK. ${ }^{19}$ As an example, the school of nursing at the University of Texas Health Service Center at Houston offers a full time ENP programme of study that lasts for 21 months and includes classroom as well as clinical learning opportunities: the Master of Science in Nursing (MSN) is awarded upon successful completion of the programme. ${ }^{20}$ Cooper and Robb feel that the UK often reflects changes in the USA and it may not be long before specific degrees at Master level are developed for nurse practitioners. ${ }^{21}$ Crouch and Jones have investigated the possibility of developing a faculty of emergency nursing. ${ }^{22}$

The Royal College of Nursing offers a BSc (Hons) Nurse Practitioner (Primary Health Care) course. This is a modular programme, each module lasting for 15 weeks. Details of other courses are available from the NHS Careers service (0845 6060655).

There can be little doubt that ENPs can provide an invaluable adjunct to the traditional role of the senior nurse in the A\&E department. It seems very likely that ENP services will become increasingly widespread throughout the UK, will become more formalised, and will expand in scope and clinical responsibility, to the mutual benefit of patients and staff.

1 Robinson S, Inyang V. The nurse practitioner in emergency medicine - valuable but undefined. Lancet 1999;354:131920.

2 Read SM, Jones NMB, Williams BT. Nurse practitioners in accident and emergency departments: what do they do? accident and emergency

3 English T. Personal paper: Medicine in the 1990s needs a team approach. BMF 1997;314: 661-3.

4 Tye CC, Ross F, Kerry SM. Emergency nurse practitioner services in major accident and emergency departments: a United Kingdom postal survey. F Accid Emerg Med 1998;15:31-4

5 Sakr M, Angus J, Perrin J, et al. Care of minor injuries by emergency nurse practitioners or junior doctors: randomised controlled trial. Lancet 1999;354:1321-6.

6 Crux S. Nurse practitioner service relieves pressure in A\&E. Nursing Times 1997;93:50-1.

7 Brebner JA, Ruddick-Bracken H, Norman JN, et al. The nurse practitioner: management of minor trauma. Accident nurse practitioner: management of min

8 Meek S, Kendall J, Porter J, et al. Can accident and emergency nurse practitioners interpret radiographs? A emergency nurse practitioners interpret radiographs?

9 Salt P, Clancy M. Implementation of the Ottawa ankle rules by nurses working in an accident and emergency department. F Accid Emerg Med 1997;14:363-5.

10 Mann CJ, Grant I, Guly H, et al. Use of the Ottawa ankle rules by nurse practitioners. F Accid Emerg Med 1998;15: $315-16$

11 Thurston J, Field F. Should accident and emergency nurses request radiographs? Results of a multicentre evaluation. $\mathcal{F}$ Accid Emerg Med 1996;13:86-9.

12 Freij RM, Duffy T, Hackett D, et al. Radiographic interpretation by nurse practitioners in a minor injuries unit. $f$ Accid Emerg Med 1996;13:41-3.

13 Marshall J, Edwards C, Lambert M. Administration of medicines by emergency nurse practitioners according to protocols in an accident and emergency department. $f$ Accid Emerg Med 1997;14:233-7.

14 Meek SJ, Ruffles G, Anderson J, et al. Nurse practitioners in major accident and emergency departments: a national survey. F Accid Emerg Med 1995;12:177-81.

15 Bache J, Armitt C, Gadd C. Practical procedures in the emergency department. London: Mosby, 1998.

16 Senior K. Emergency nurse practitioner. Emergency Nurse 1999:6:28-32.

17 Mabrook AF, Dale B. Can nurse practitioners offer a quality service? An evaluation of a year's work of a nurse led minor injury unit. F Accid Emerg Med 1998;15:266-8.

18 Orzel MN. A collaborative generic curriculum development to support practitioners functioning within enhanced roles. Accident and Emergency Nursing 1998;6:149-54.

19 Tye CC. The emergency nurse practitioner role in major accident and emergency departments: professional issues accident and emergency departments: professional iss
and the research agenda. $\mathcal{f}$ Adv Nurs 1997;26:364-70.

20 Cole F, Ramirez E, Mickanin J. ENP education: a United States perspective. Emergency Nurse 1998;6:12-14.

21 Cooper M, Robb A. Nurse practitioners in A\&E: a literature review. Emergency Nurse 1996;4:19-22.

22 Crouch R, Jones GJ. Towards a faculty of emergency nursing: planning for the future. Emergency Nurse 1997;5: $12-15$. 